\title{
Effects of an intravitreal injection of interleukin-35-expressing plasmid on pro-inflammatory and anti-inflammatory cytokines
}

\author{
CHAO HOU, QIANNI WU, CHEN OUYANG and TING HUANG \\ State Key Laboratory of Ophthalmology, Zhongshan Ophthalmic Center, Sun Yat-sen University, \\ Guangzhou, Guangdong 510030, P.R. China
}

Received November 30, 2015; Accepted June 27, 2016

DOI: $10.3892 / \mathrm{ijmm} .2016 .2688$

\begin{abstract}
In order to explore the potential effects of interleukin (IL)-35 on IL-10, transforming growth factor- $\beta$ (TGF- $\beta$ ), interferon- $\gamma$ (INF)- $\gamma$, IL-12 and IL-17, a pcDNA3.1-IL-35 plasmid was injected into the vitreous cavity of BALB/c mice. Enzyme-linked immunosorbent assay, western blot analysis and quantitative PCR analysis were performed to confirm the successful expression of IL-35. Slit-lamp biomicroscopy, hematoxylin and eosin staining and immunofluorescence were employed to detect the status of eyes, and western blot analysis was performed to examine the expression of corneal graft rejection-related cytokines. There were no abnormalities in the eyes pre-mydriasis or post-mydriasis and no injuries to the cornea or retina following the injection of IL-35-expressing plasmid. An immunofluorescence assay detected the positive expression of IL-35 in corneal epithelial cells from IL-35-injected mice and negative staining in the control group. Further study revealed that IL-35 enhanced the expression of IL-10 and TGF- $\beta$ which reached their highest levels at 1 and 2 weeks after injection, respectively $(p<0.01)$. Moreover, the expression of INF- $\gamma$ and IL-12 was decreased significantly at 2 weeks after the injection of IL-35-expressing plasmid ( $\mathrm{p}<0.05$ ), and the expression of IL-17 was suppressed notably at 4 weeks after the injection $(\mathrm{p}<0.05)$. The intravitreal injection of IL-35-expressing plasmid in mice downregulates the expression of pro-inflammatory cytokines and upregulates the expression of anti-inflammatory cytokines. Thus, IL-35 may further be assessed as a potential target for the treatment of corneal graft rejection.
\end{abstract}

\section{Introduction}

The cornea is an immunologically privileged area, and keratoplasty is one of the most successful forms of solid tissue

Correspondence to: Professor Ting Huang, State Key Laboratory of Ophthalmology, Zhongshan Ophthalmic Center, Sun Yat-sen University, 54 South Xianlie Road, Yuexiu, Guangzhou, Guangdong 510030, P.R. China

Email: thuang@vip.163.com

Key words: interleukin-35, intravitreal injection, inflammatory cytokines, corneal graft rejection transplantation that serves as the only clinical therapy for blindness (1). Approximately 36,000 cases of corneal transplantation surgery are performed annually in the USA and about 3,000 cases in China each year $(2,3)$. Despite steady improvements in the graft survival rate, immune-mediated graft rejection remains the major cause of human corneal allograft failure, particularly following high-risk keratoplasty or a second keratoplasty $(4,5)$. Currently, glucocorticoids, cyclosporin A and tacrolimus are the main clinical treatment strategies for corneal graft rejection; however, treatment is limited by the non-specific inhibition of immune defense function. Hence, specific local therapeutic approaches are expected to prolong the survival time of corneal grafts.

$\mathrm{CD}^{+} \mathrm{T}$-cells play an important role in corneal graft rejection (6). Activated naive $\mathrm{CD}^{+}{ }^{+} \mathrm{T}$-helper cells (Th0) differentiate into Th1, Th2 or Th3 cell subsets depending on the different kinds of cytokines within the graft microenvironment. The Th cells were then activated by recognizing alloantigens to secrete a series of cytokines including IL-10, interferon- $\gamma$ (INF- $\gamma$ ) and transforming growth factor- $\beta$ (TGF- $\beta$ ) (7).

Interleukin (IL)-35, a heterodimer composed of p35 and Epstein-Barr virus-induced gene 3 (EBI3) subunits, belongs to the IL-12 family and exerts anti-inflammatory and immunomodulatory effects (8). It is predominantly expressed by thymus-derived and peripheral $\mathrm{CD} 4{ }^{+} \mathrm{CD} 25^{+} \mathrm{Foxp} 3^{+}$regulatory T (Treg) cells (9). Previous findings have demonstrated that the elevated serum IL-35 level was associated with the inflammatory response in multiple inflammatory diseases such as acute pancreatitis (10), collagen-induced arthritis (CIA) (11), periodontal inflammation (12), allergic rhinitis and asthma (13) as well as coronary artery disease. In vitro studies demonstrated that animals without functional IL-35 exhibited enhanced inflammatory immune responses and were more likely to develop diseases, such as liver fibrosis, inflammatory bowel disease and models of lethal autoimmune disease (14-17). Furthermore, reduced IL-35 levels are associated with rejection following allogeneic hematopoietic stem cell transplantation (10), and IL-35 therapy may inhibit cardiac allograft rejection in mice (18). Jin et al demonstrated that the expression of IL-35 in human placental trophoblasts may prevent matrix immune rejection induced by fetal antigens (19). However, the effect of IL-35 on other corneal graft rejection-related cytokines in the eyes has not been substantiated. 
In the present study, we successfully injected a pcDNA3.1-IL-35 plasmid into the mouse vitreous cavity to observe whether IL-35 affected the expression of corneal graft rejection-related cytokines. Our results showed that intravitreal injection of pcDNA3.1-IL-35 plasmid is safe for mouse eyes. Furthermore, enhanced levels of IL-35 may suppress pro-inflammatory cytokine expression and increase anti-inflammatory cytokine expression.

\section{Materials and methods}

Animals. A total of 72 specific pathogen-free (SPF) female BALB/c mice, aged 6-10 weeks old and weighing between 15-18 $\mathrm{g}$ were purchased from the Medical Laboratory Animal Center of Sun Yat-sen University (Guangzhou, China). This study was approved by the Ethics Committee of Sun Yat-sen University. All animal experiments were performed in accordance with the Guidelines of Institutional Animal Care and Use Committee at Sun Yat-sen University.

Intravitreal injection of pcDNA3.1-IL-35 plasmid. The pcDNA3.1-IL-35plasmidharboringIL-35-codingsequenceswas constructed by Guangzhou Vipotion Biotechnology Co., Ltd. (Guangzhou, China). Each mouse was deeply anesthetized by an intraperitoneal injection of $4.3 \%$ chloral hydrate (China National Medicines Corporation, Ltd., Beijing, China) and mydriasis was induced with tropicamide eye drops (Shenyang Xingqi Pharmaceutical Co., Ltd., Shenyang, China). To induce superficial anesthesia of the eye, $0.5 \%$ tetracaine hydrochloride (National Institutes for Food and Drug Control, Beijing, China) was subsequently used. A $33 \mathrm{~g}$ Hamilton microinjector was used to puncture the vitreous cavity at a $45^{\circ}$ angle to the transection of the lens and $1 \mu \mathrm{l}(1 \mu \mathrm{g} / \mu \mathrm{l})$ pcDNA3.1-IL-35 plasmid was injected into vitreous cavity. Subsequently, tobramycin (Qilu Pharmaceutical Co., Ltd., Jinan, China) was applied to the conjunctival sac. Equal volumes of pcDNA3.1 plasmid and PBS were injected in parallel as a control. Ocular status (pre-mydriasis and post-mydriasis) was examined by slit-lamp biomicroscopy 1,2 and 4 weeks after injection and 6-8 mice in each group were then sacrificed by cervical dislocation. The mouse eyes were enucleated for subsequent experiments.

Enzyme-linked immunosorbent assay (ELISA). The enucleated eyes in each mouse were lysed according to a previously published method (20). Thereafter, ELISA was peformed using a mouse ELISA kit for IL-35 (USCN Business Co., Ltd., Wuhan, China) strictly according to the manufacturer's instructions, with five replicates for each testing point including blank wells and untreated controls (0 week). Optical density (OD) values were assessed at $450 \mathrm{~nm}$ by a microplate reader (Biotek, Winooski, VT, USA).

Western blot analysis. The enucleated eyes from each mouse were lysed and the total proteins were extracted as previously described (20), and the protein concentration was quantified using a bicinchoninic acid (BCA) protein assay kit (Beyotime Institute of Biotechnology, Haimen, China). Proteins $(40 \mu \mathrm{g})$ in each sample were loaded and separated using sodium dodecyl sulphate-polyacrylamide gel electrophoresis (SDS-PAGE) and transferred onto polyvinylidene fluoride (PVDF) membranes
(Millipore, Bedford, MA, USA). The membranes were then blocked with 5\% non-fat milk overnight and probed with anti-p35 (ab66064), anti-IL-10 (ab134742), anti-TFG- $\beta$ (ab190503), anti-IFN- $\gamma$ (ab24324), anti-IL-12 (ab89889) and anti-IL-17 (ab77171) primary antibodies (1:500 dilution; all from Abcam, Cambridge, MA, USA) at $4^{\circ} \mathrm{C}$ overnight, followed by incubation with secondary anti-rabbit IgG-HRP antibody (A0208) or anti-mouse IgG-HRP antibody (A0216) (1:5000 dilution; Beyotime Institute of Biotechnology) at $37^{\circ} \mathrm{C}$ for 45 min after washing twice with TBST. The target bands were visualized using enhanced chemiluminescence (ECL) solution (Qihai Biotec, Shanghai, China) and analyzed using Gel-ProAnalyzer software (Media Cybernetics, Bethesda, MD, USA). $\beta$-actin served as an internal control.

Reverse transcription-quantitative polymerase chain reaction $(R T-q P C R)$. The total RNA was extracted from the enucleated eyes using an RNA extraction kit (Tiangen Biotech, Beijing, China) and then reverse transcribed into cDNA. RT-qPCR reactions were performed in an Exicycler ${ }^{\mathrm{TM}} 96$ (Bioneer, Daejeon, Korea) using SYBR-Green Master mix (Solarbio, Beijing, China) according to the following protocol: initial denaturation at $95^{\circ} \mathrm{C}$ for $5 \mathrm{~min}, 30$ cycles consisting of $95^{\circ} \mathrm{C}$ for $20 \mathrm{sec}, 60^{\circ} \mathrm{C}$ for $20 \mathrm{sec}$, and $72^{\circ} \mathrm{C}$ for $30 \mathrm{sec}$, and final elongation at $4^{\circ} \mathrm{C}$ for $5 \mathrm{~min}$. The primer sequences were as follows: p35, 5'-GACCTGGACCCTGAGATTGTGAA-3' (sense) and 5'-GGTCCCTGTGCAGCACGTTA-3' (antisense); and $\beta$-actin, 5'-GGAGATTACTGCCCTGGCTCCTA-3' (sense) and 5'-GACTCATCGTACTCCTGCTTGCTG-3' (antisense). Relative mRNA expression was calculated using the $2^{-\Delta \Delta C T}$ method and $\beta$-actin was used as an internal control.

Hematoxylin and eosin $(H \& E)$ staining. Corneal and retinal tissues were fixed in $10 \%$ formaldehyde for $24 \mathrm{~h}$, dehydrated with a graded series of ethanol $(70 \%$ for $2 \mathrm{~h}, 80 \%$ overnight, $90 \%$ for $2 \mathrm{~h}$ and $100 \%$ for $2 \mathrm{~h}$ ) and embedded in dimethylbenzene-paraffin. Sections $(5 \mu \mathrm{m})$ were cut, placed onto slides and dried in a $70^{\circ} \mathrm{C}$ chamber for $40 \mathrm{~min}$. After being dewaxed with ethanol, the sections were soaked with hematoxylin (Solarbio), water and $1 \%$ hydrochloric ethanol at room temperature for $5 \mathrm{~min}, 5 \mathrm{~min}$, and $3 \mathrm{sec}$ respectively, and rinsed with water for $20 \mathrm{~min}$. The slices were subsequently stained with eosin for 3 min, and dehydrated through a graded series of ethanol (75, $85,95 \%$ each for $2 \mathrm{~min}$, and $100 \%$ twice for $5 \mathrm{~min}$ ). The stained tissues were then permeabilized twice with xylene for $10 \mathrm{~min}$ and mounted with resinene and observed under a microscope (DP73; Olympus, Tokyo, Japan) and images were captured.

Immunofluorescence double staining. The $5 \mu \mathrm{m}$ slices were fixed in $4 \%$ formaldehyde for $15 \mathrm{~min}$, washed with PBS three times, and blocked with $10 \%$ bovine serum albumin (BSA) at room temperature for $1 \mathrm{~h}$. Thereafter, the cells were co-incubated with p35 (sc-821; 1:50 dilution; Santa Cruz Biotechnology, Santa Cruz, CA, USA) and EBI3 primary antibodies (sc-166158; 1:250 dilution; Santa Cruz Biotechnology) at $4^{\circ} \mathrm{C}$ overnight and subsequently incubated with FITClabeled goat anti-rabbit $\operatorname{IgG}(\mathrm{H}+\mathrm{L})(\mathrm{A} 0562)$ and $\mathrm{Cy} 3$-labeled goat anti-mouse $\mathrm{IgG}(\mathrm{H}+\mathrm{L})$ (A0516) secondary antibodies (Beyotime Institute of Biotechnology) at a dilution of 1:200 for $1 \mathrm{~h}$ at room temperature. Unbound antibodies in each step 
A

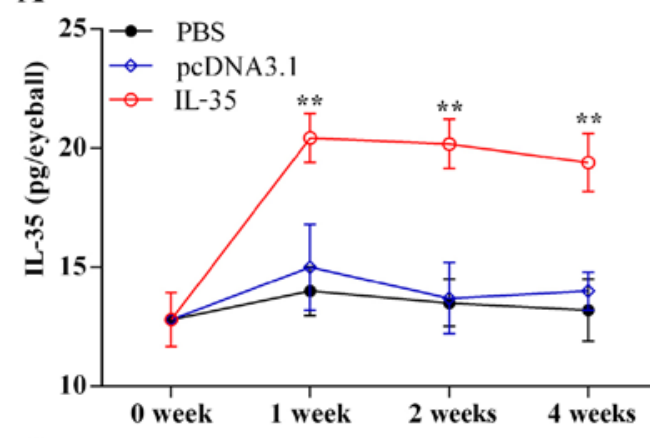

C

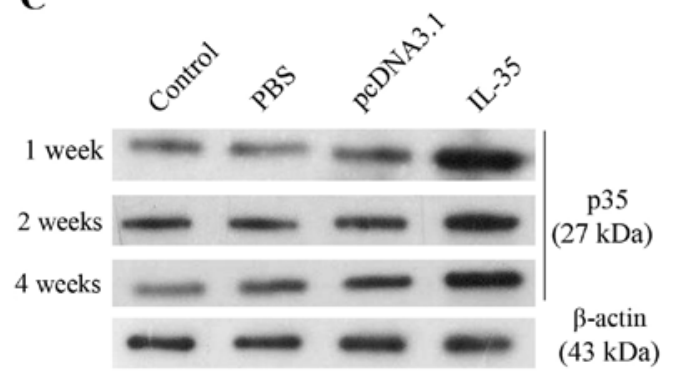

B

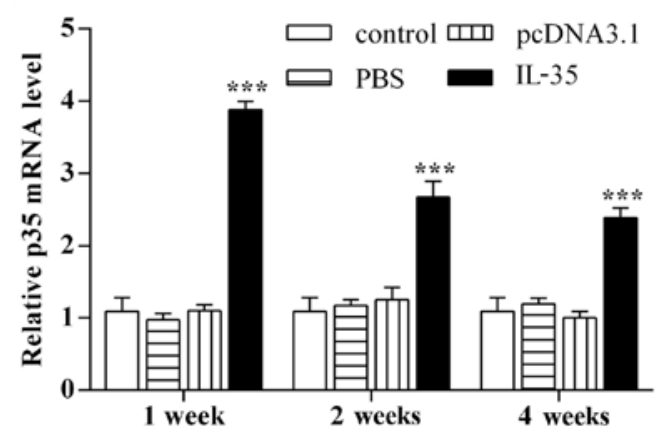

D

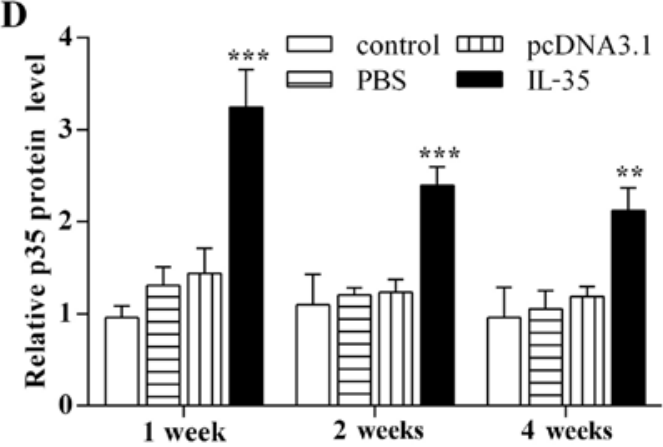

Figure 1. Intravitreal injection of pcDNA3.1-interleukin (IL)-35 plasmid increases the intraocular level of IL-35. The plasmid pcDNA3.1-IL-35 encoding IL-35 sequences was injected into the vitreous cavity of mouse eyes. Mice were raised for 1, 2 and 4 weeks, respectively, prior to sacrifice. Thereafter, the mouse eyes were enucleated and lysed. (A) Enzyme linked immunosorbent assay (ELISA) was employed to examine the secretion level of IL-35 with five replicates at each testing point. (B) RT-qPCR analysis of p35 mRNA levels. $\beta$-actin was used as an internal control. Six to eight mice were randomly selected in each group and experiments were performed in triplicate for statistical significance. (C) Representative images of western blot analysis of p35 protein levels. $\beta$-actin served as the internal control. (D) The relative expression of $\mathrm{p} 35$ protein normalized to $\beta$-actin. The results are expressed as the means \pm standard deviation (SD). ${ }^{* *} \mathrm{p}<0.01$, ${ }^{* * *} \mathrm{p}<0.001 \mathrm{vs}$. pcDNA3.1.

were removed by washing with PBS. The coverslips were mounted inversely onto slides with $95 \%$ glycerol and observed under a laser scanning confocal microscope (Olympus) and images were captured.

Statistical analysis. Statistical analysis was performed using GraphPad Prism 5.0 software. All data are reported as the means \pm standard deviation (SD). The differences between groups were analyzed by one-way analysis of variance (ANOVA). A p-value $<0.05$ was considered to indicate a statistically significant difference.

\section{Results}

Intravitreal injection of pcDNA3.1-IL-35 plasmid increases intraocular level of $I L-35$. To explore the function of IL-35 in the eyes of mice, the pcDNA3.1-IL-35 plasmid encoding IL-35 was injected into the vitreous cavity of BALB/c mice, and then the expression level of IL-35 was detected by ELISA, RT-qPCR and western blot analysis. The results of the ELISA showed that the intraocular IL-35 level was significantly elevated at 1 week after injection with the pcDNA3.1-IL-35 plasmid compared with pcDNA3.1 injection (Fig. 1A; p<0.01) and in fact, peaked after 1 week $(20.43 \pm 1.032 \mathrm{pg}$ /eyeball). Moreover, the mRNA levels of intraocular p35 at 1,2 and 4 weeks after injection with the pcDNA3.1-IL-35 plasmid were 3.53-, 2.39 and 2.14-fold (Fig. 1B; p<0.001) higher, respectively, than those injected with with respective pcDNA3.1. The protein levels of intraocular p35 at 1, 2 and 4 weeks after IL-35 plasmid injection were 2.25-, 1.94- and 1.79-fold higher, respectively, (Fig. 1B and $\mathrm{C} ; \mathrm{p}<0.01$ ) than those in the presence of pcDNA3.1 alone. These data suggest the successful expression of the pcDNA3.1-IL-35 plasmid in the mouse eyes.

Intravitreal injection of pcDNA3.1-IL-35 plasmid is safe to ocular tissues. To determine the effect of an intravitreal injection of pcDNA3.1-IL-35 plasmid on intraocular tissues, slit-lamp and $H \& E$ staining were employed to evaluate the physiological changes in the cornea and retina. As shown in Fig. 2A, there were no differences between the mouse eyes injected with pcDNA3.1IL-35 plasmid and the control. The cornea exhibited transparency without obvious edema, the aqueous humor was clear, iris vessels were clearly visible, and the size and location of the pupil was normal. There was no intraocular hemorrhage, retinal detachment, or neovascularization during the 4 week period following plasmid injection. Further H\&E staining revealed no abnormalities of the cornea in all groups except for the loose arrangement of corneal stroma collagen fibers which disappeared at 4 weeks after injection (Fig. 2B). There were no apparent abnormalities in the retinal pigment epithelium, retinal cells, cell layer or bipolar cells, indicating that the method of intravitreal injection of pcDNA3.1-IL-35 plasmid is safe to ocular tissues.

IL-35 is uniformly distributed on corneal epithelial cells. IL-35 is formed by pairing IL-12 $\alpha$ (also known as p35) and EBI3. As IL-35 shares the p35 subunit with IL-12 and shares the EBI3 subunit with IL-27 (8), we employed the immunofluorescence double staining assay to identify the distribution of intraocular IL-35. As shown in Fig. 3, there 
A

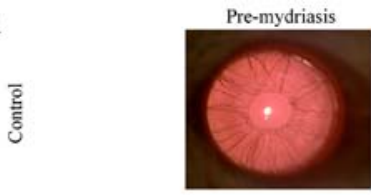

Pre-mydriasi
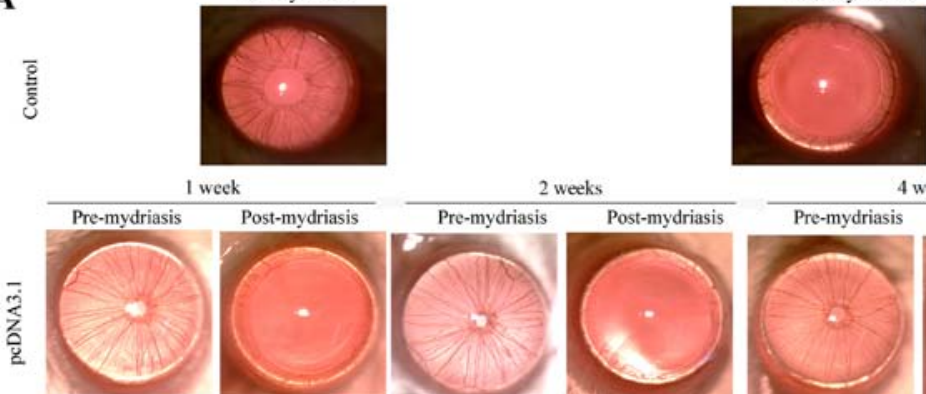

Post-mydriasis

2 weeks

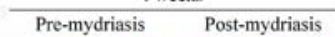

$\stackrel{3}{=}$
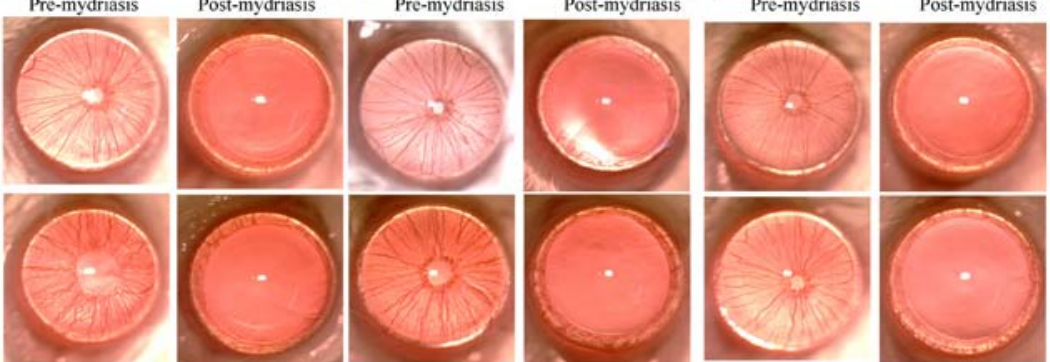

$\mathbf{B}$
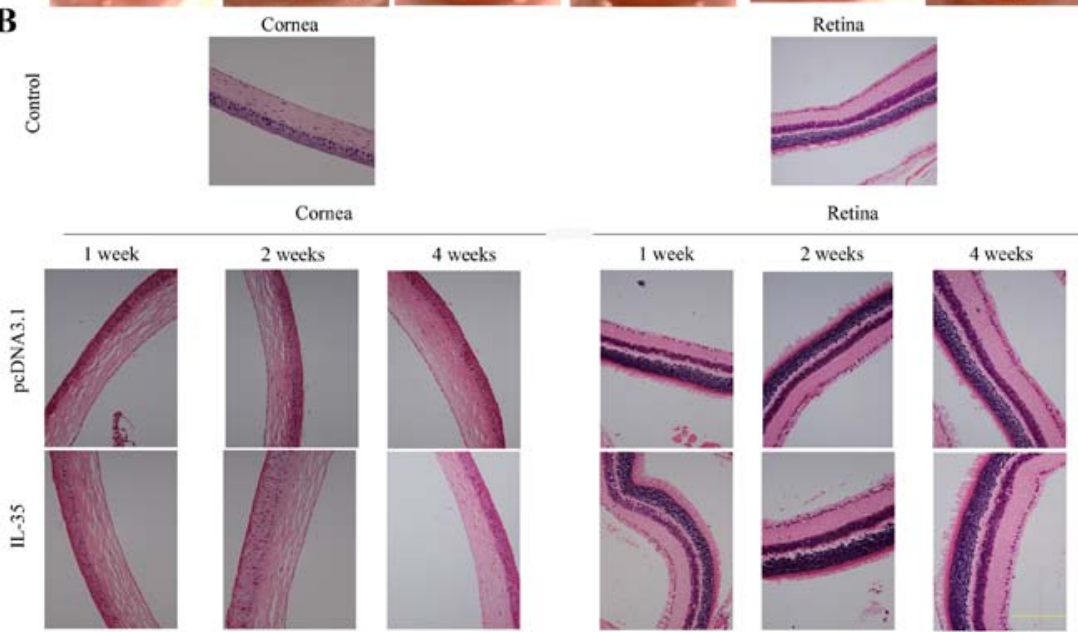

Figure 2. Intravitreal injection of pcDNA3.1-interleukin (IL)-35 plasmid is safe in ocular tissues. (A) Ocular status of mouse eyes pre-mydriasis and post-mydriasis at the indicated time periods were observed by slit-lamp biomicroscopy. Representative examples of images are shown. (B) Representative photomicrographs of corneal and retinal tissues with or without IL-35 plasmid intravitreal injection. Nuclei labeled with hematoxylin were observed to be blue and cytoplasm stained with eosin was red. Scale bar, $50 \mu \mathrm{m}$. The above two experiments were repeated three times.
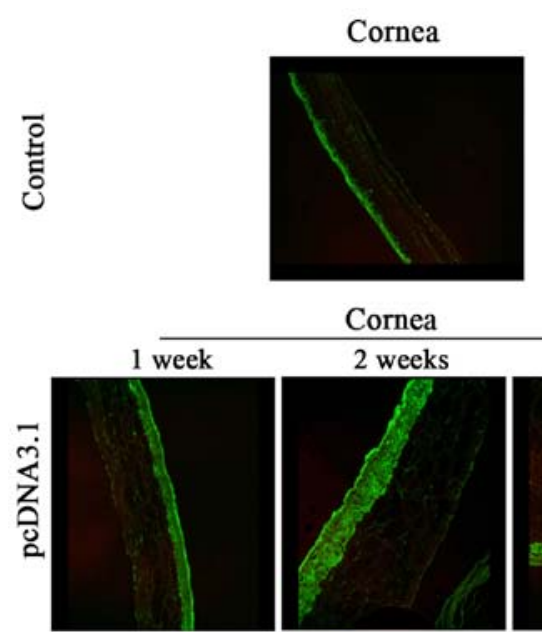

Cornea
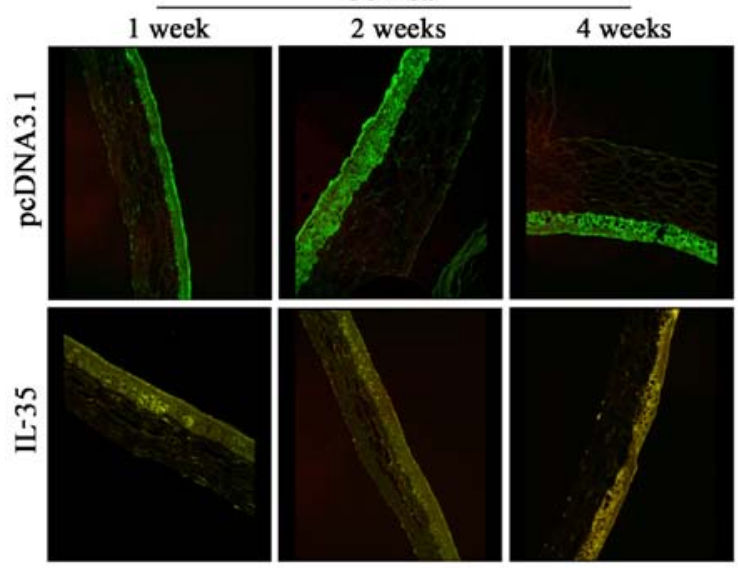

Figure 3. Interleukin (IL)-35 is uniformly distributed in corneal epithelial cells. An immunofluorescence double assay was performed to examine the distribution of IL-35 in the cornea and retina. The representative merged images were selected from three independent experiments. p35 was visualized using FITC-labeled goat anti-rabbit IgG $(\mathrm{H}+\mathrm{L})$ as green and EBI3 was stained with Cy3-labeled goat anti-mouse $\mathrm{IgG}(\mathrm{H}+\mathrm{L})$ as red. Scale bar, $50 \mu \mathrm{m}$. 
A

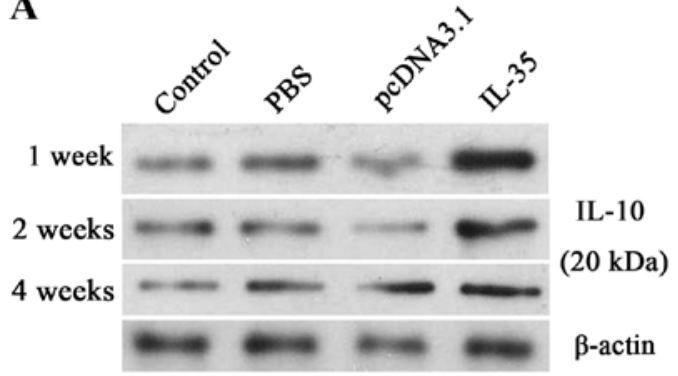

(43 kDa)

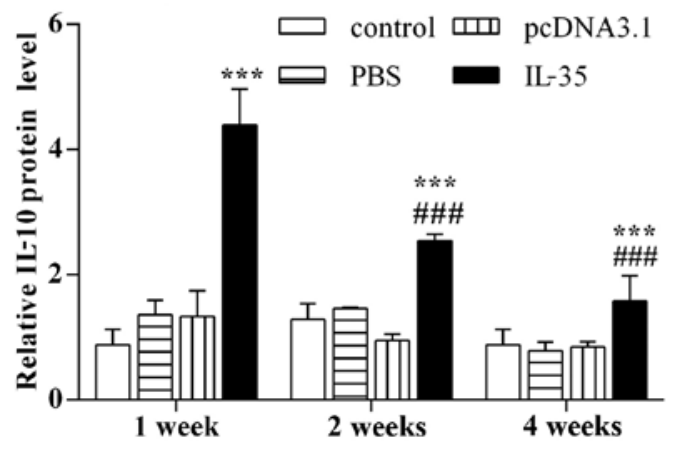

B

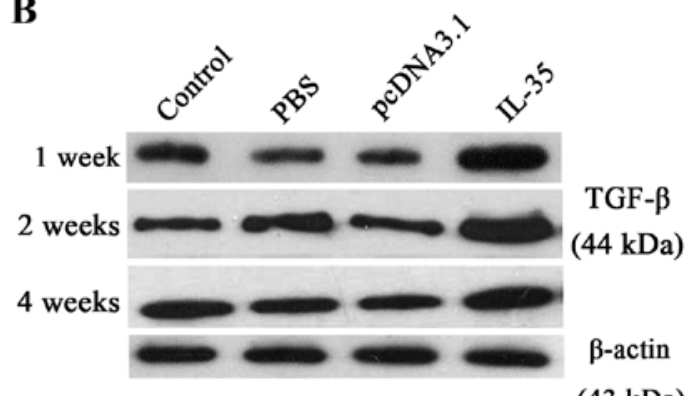

(43 kDa)

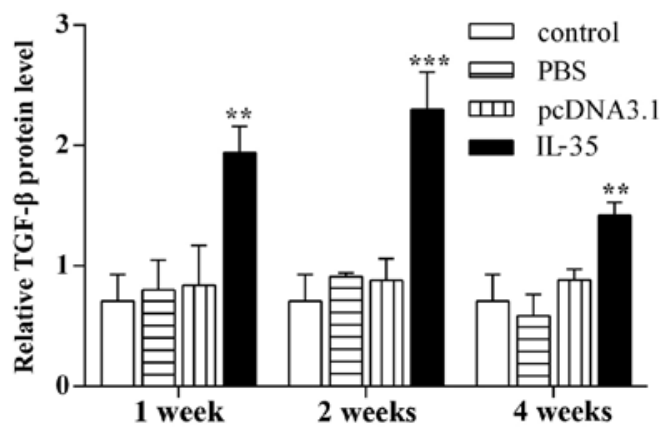

Figure 4. Elevated interleukin (IL)-35 levels enhance the expression of intraocular IL-10 and transforming growth factor- $\beta$ (TGF- $\beta$ ). Western blot analysis was performed to evaluate the protein expression of (A) IL-10 and (B) TGF- $\beta$ at different time-points following an intravitreal injection of pcDNA3.1-IL-35 plasmid. Representative blots are shown and corresponding densitometric analysis is presented as the means \pm standard deviation (SD) from three independent experiments. $\beta$-actin was used as the internal control. ${ }^{* *} \mathrm{p}<0.01,{ }^{* * * *} \mathrm{p}<0.001 \mathrm{vs}$. control; ${ }^{\# \# \#} \mathrm{p}<0.001$ vs. 1 week of IL-35.

was no IL-35 expression in the corneal epithelial cells of the control- or pcDNA3.1-injected mice, as evidenced by the positively stained p35 antibody and negatively stained EBI3 antibody. Moreover, we observed coexpressed p35 (green) and EBI3 (red) on corneal epithelial cells from the pcDNA3.1-IL-35 plasmid-injected mice, and their localizations were matched, indicating the successful expression of IL-35, and that the expression of IL-35 in the corneal epithelial cells lasted for at least 4 weeks. On the other hand, the expression of IL-35 was predominantly distributed in the retinal epithelium in both the control and pcDNA3.1-IL-35 plasmid-injected mice. The above results demonstrate that IL-35 is expressed on mouse corneal epithelial cells following intravitreal injection with the pcDNA3.1-IL-35 plasmid.

Elevated IL-35 level enhances the expression of intraocular $I L-10$ and $T G F-\beta$. We conducted western blot analysis to examine the effect of IL-35 on the expression of anti-inflammatory cytokines. The results showed that the expression of IL-10 was notably enhanced by 5.0 -fold compared with the control 1 week following an intravitreal injection of pcDNA3.1-IL-35 plasmid (Fig. 4A; p<0.001). The increased IL-10 level then faded markedly at 2 and 4 weeks after injection $(p<0.001)$. Moreover, an intravitreal injection of pcDNA3.1-IL-35 plasmid resulted in a significant increase of TGF- $\beta$ expression (Fig. 4B; $p<0.01$ ), which reached a peak at 2 weeks $(\mathrm{p}<0.001)$. The above results indicate that an intravitreal injection of pcDNA3.1-IL-35 plasmid in mice upregulates the expression of anti-inflammatory cytokines.

Elevated IL-35 level suppresses the expression of INF- $\gamma, I L-12$ and $I L-17$. Western blot analysis was performed to observe the effect of IL-35 on the expression of pro-inflammatory cytokines. The results showed that an intravitreal injection of pcDNA3.1-IL-35 plasmid induced a sharp decline in the expression of INF- $\gamma 2$ weeks after injection (Fig. 5A; p<0.05). Simultaneously, the expression of IL-12 was apparently decreased by 1.91 -fold compared with the control at 2 weeks after the pcDNA3.1-IL-35 plasmid injection (Fig. 5B; p<0.05). In addition, a marked downregulation of IL-17 was observed at 4 weeks after the pcDNA3.1-IL-35 plasmid injection (Fig. 5C; $\mathrm{p}<0.05)$. Taken together, these findings demonstrate that elevated IL-35 levels inhibit the expression of pro-inflammatory cytokines.

\section{Discussion}

The anti-inflammatory cytokine IL-35 is able to inhibit rejection following organ transplantation. However, the role of IL-35 in corneal graft rejection remains unclear, and the effect of IL-35 on corneal graft rejection-related cytokines has not been fully elucidated. Herein, we found that increased IL-35 expression promoted the expression of anti-inflammatory cytokines and reduced the expression of pro-inflammatory cytokines following an intravitreal injection of pcDNA3.1-IL-35 plasmid, which was also proved to be safe to use in ocular tissues as there were no abnormalities observed in the mouse corneal and retinal tissues. Collectively, these findings demonstrate that an intravitreal injection of plasmid harboring IL-35 may be a potential approach for reducing the risk of corneal allograft rejection.

The immunosuppressive effect of IL-35 is not only characterized by the suppression of effector $\mathrm{T}$ cell (Teff) responses and Teff proliferation, but is also manifested in the induction of 

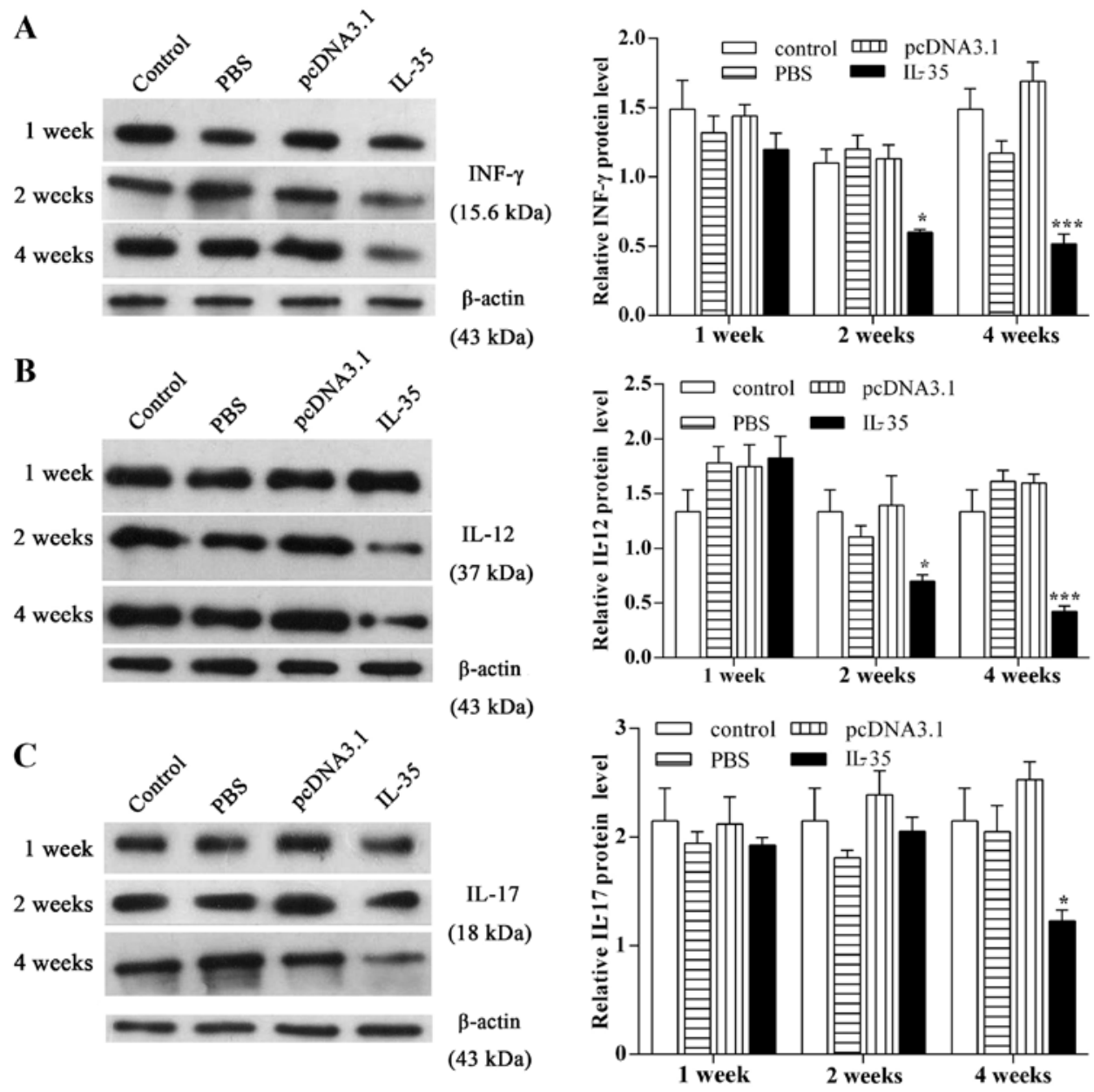

Figure 5. Elevated interleukin (IL)-35 levels suppress the expression of interferon- $\gamma$ (INF- $\gamma$ ), IL-12 and IL-17. The protein expression of (A) INF- $\gamma$, (B) IL-12 and (C) IL-17 were detected by western blot analysis and normalized to $\beta$-actin. Representative examples of images are shown and the data are presented as the means \pm standard deviation (SD), and the error bars represent the $\mathrm{SD}$ of three independent experiments. ${ }^{*} \mathrm{P}<0.05,{ }^{* * *} \mathrm{P}<0.001$ vs. control.

Tregs to generate and propagate infectious tolerance, and these Tregs in turn secrete IL-35 to enhance the immunosuppressive effect $(14,15,21)$. Detailed studies have identified that Tregs play an essential role in protecting individuals against graft rejection and prolonging survival time, including corneal transplantation $(22,23)$. In view of these findings, we hypothesized that IL-35 may contribute to the inhibition of corneal allograft rejection. Moreover, plasmid injection does not result in genomic integration, therefore the expression of foreign genes in the tissue cells is temporary, and the cells will gradually lose the plasmid over time (24). Previous studies examined the hydrodynamic tail vein injection of plasmid DNA; however, efficient expression was only detected in the mouse liver (25-27). Other studies found that an intravitreal injection of plasmid DNA led to expression in the cornea (28-30). Thus, we preliminarily detected alterations in the expression of relevant cytokines after injecting the pcDNA3.1-IL-35 plasmid into the vitreous cavity of mice. We detected that the expression of IL-35 lasted for at least 4 weeks in corneal epithelial cells, which is concurrent with the occurrence time of an alloantigen-induced inflammatory reaction following corneal allograft surgery.

IL-10 and TGF- $\beta$ are secreted by a variety of cell types with graft tolerance and anti-inflammatory properties (31). IL-10 may block the production of IL-12 and downregulate
MHC class II expression on monocytes (32). TGF- $\beta$ regulates the proliferation, differentiation and apoptosis of several immune cells (33). Jin et al proved that IL-35 boosted the proliferation of Tregs by increasing the expression of IL-10 and TGF- $\beta$, which was important for the establishment and maintenance of maternal-fetal tolerance during early pregnancy (19). In this study, we demonstrated that the expression of IL-10 and TGF- $\beta$ were significantly increased in the eyes following an intravitreal injection of pcDNA3.1-IL-35 plasmid, which was consistent with the previous findings of Jin et al. The elevated levels of IL-10 and TGF- $\beta$ are closely associated with immune tolerance to the corneal allograft (34-36). Klebe et al reported that transferred ovine IL-10-cDNA reduced the incidence of corneal graft rejection and prolonged corneal allograft survival (37). Wang et al found that TGF- $\beta$ plays an important role in the conversion of Tregs from T-helper (Th)17 cells and thereby affects the Treg-Th17 balance to facilitate immunological tolerance following allogenic corneal transplantation (38). Hence, we demonstrated that the exogenous injection of IL-35 upregulated the expression of the graft tolerance-related cytokines, IL-10 and TGF- $\beta$.

INF- $\gamma$ is a potent, pro-inflammatory cytokine responsible for strengthening the Th1 immune response, and IL-12 is 
another pro-inflammatory cytokine capable of promoting the proliferation of Th1-type cells (39). Recent evidence suggested that IL-12 stimulated IFN- $\gamma$ production by NK and $\mathrm{T}$ cells (40), and the increased production of IL-12 and IFN- $\gamma$ may be predictive of corneal graft rejection $(41,42)$. In our study, we detected the downregulation of IL-12 and IFN- $\gamma$ in pcDNA3.1-IL-35 plasmid-injected eyes, indicating the presence of an anti-inflammatory environment around the cornea. Additionally, IL-17, a potent pro-inflammatory cytokine, mediates tissue inflammation by inducing chemokine expression and leukocyte infiltration (43). The IL-17 antagonist prolonged the median survival time of non-vascularized and vascularized cardiac allograft patients and improved the survival rate of corneal allogeneic transplantation (44-46). Chen et al observed that the anti-IL-17 mAb suppressed the expression of IFN- $\gamma$ and IL-12 p40 significantly in transplanted splenocytes $(46,47)$. Similarly, we found a notable decline in IL-17 expression 4 weeks after IL-35 injection and more significantly reduced IFN- $\gamma$ and IL-12 levels at the same time point, suggesting that the decreased expression of IL-17 may further reduce IFN- $\gamma$ and IL-12 levels. Taken together, these findings demonstrated that an intravitreal injection of pcDNA3.1-IL-35 plasmid downregulated the expression of the pro-inflammatory cytokines IL-12, IL-17 and IFN- $\gamma$.

There are some limitations in our study. We explored the effect of elevated IL-35 expression on corneal allograft rejection-related cytokines in healthy mice without establishing a model of corneal allograft rejection. In our next study, we will detect IL-35 distribution and pathological changes in other ocular tissues following an intravitreal injection of pcDNA3.1-IL-35 plasmid in a murine model of corneal allograft transplantation.

The findings of the present study suggest that an intravitreal injection of IL-35 plasmid suppresses the expression of pro-inflammatory cytokines and promotes the expression of anti-inflammatory cytokines. Our data preliminarily identified the effects of IL-35 on corneal allograft rejection-related cytokines, and suggest that IL-35 may serve as a potential target for gene therapy of corneal allograft rejection.

\section{Acknowledgements}

The present study was supported by the National Natural Science Foundation of China (no. 81371065).

\section{References}

1. Qin Q, Shi Y, Zhao Q, Luo D, Chen Y, Wu J and Zhao M: Effects of CD25siRNA gene transfer on high-risk rat corneal graft rejection. Graefes Arch Clin Exp Ophthalmol 253: 1765-1776, 2015.

2. Pan Q, Xu Q, Boylan NJ, Lamb NW,Emmert DG, Yang JC, Tang L, Heflin T, Alwadani S, Eberhart CG, et al: Corticosteroid-loaded biodegradable nanoparticles for prevention of corneal allograft rejection in rats. J Control Release 201: 32-40, 2015.

3. Chen Y, Liao C, Gao M, Belin MW, Wang M, Yu H and Yu J: Efficacy and safety of corneal transplantation using corneas from foreign donors versus domestic donors: a prospective, randomized, controlled trial. J Ophthalmol 2015: 178289, 2015.

4. Paunicka KJ, Mellon J, Robertson D, Petroll M, Brown JR and Niederkorn JY: Severing corneal nerves in one eye induces sympathetic loss of immune privilege and promotes rejection of future corneal allografts placed in either eye. Am J Transplant 15: $1490-1501,2015$
5. Zhang H, Wang L and Zhang L: Cyclosporine nanomicelle eye drop: a novel medication for corneal graft transplantation treatment. Biol Pharm Bull 38: 893-900, 2015.

6. Pleyer U, Milani JK, Dukes A, Chou J, Lutz S, Rückert D, Thiel HJ and Mondino BJ: Effect of topically applied anti-CD4 monoclonal antibodies on orthotopic corneal allografts in a rat model. Invest Ophthalmol Vis Sci 36: 52-61, 1995.

7. Niederkorn JY: Immunology and immunomodulation of corneal transplantation. Int Rev Immunol 21: 173-196, 2002.

8. Olson BM, Sullivan JA and Burlingham WJ: Interleukin 35: a key mediator of suppression and the propagation of infectious tolerance. Front Immunol 4: 315, 2013.

9. Käser T, Müllebner A, Hartl RT, Essler SE, Saalmüller A and Catharina Duvigneau J: Porcine T-helper and regulatory T cells exhibit versatile mRNA expression capabilities for cytokines and co-stimulatory molecules. Cytokine 60: 400-409, 2012.

10. Zhang YL, Zhou XY, Guo XY and Tu JW: Association between serum interleukin-35 levels and severity of acute pancreatitis. Int J Clin Exp Med 8: 7430-7434, 2015.

11. Filková M, Vernerová Z, Hulejová H, Prajzlerová K, Veigl D, Pavelka K, Vencovský J and Šenolt L: Pro-inflammatory effects of interleukin-35 in rheumatoid arthritis. Cytokine 73: 36-43, 2015.

12. Köseoğlu S, Sağlam M, Pekbağrıyanık T, Savran L and Sütçü R: Level of interleukin-35 in gingival crevicular fluid, saliva, and plasma in periodontal disease and health. J Periodontol 86: 964-971, 2015.

13. Ding LF, Chen Q, Li L, Liu JM, Zhang GP, Zhu XH, Wu AM, Ke JW, Dai YL and Wu CX: Effects of sublingual immunotherapy on serum IL-17 and IL-35 levels in children with allergic rhinitis or asthma. Zhongguo Dang Dai Er Ke Za Zhi 16: 1206-1210, 2014 (In Chinese).

14. Collison LW, Chaturvedi V, Henderson AL, Giacomin PR, Guy C, Bankoti J, Finkelstein D, Forbes K, Workman CJ, Brown SA, et al: IL-35-mediated induction of a potent regulatory T cell population. Nat Immunol 11: 1093-1101, 2010.

15. Collison LW, Workman CJ, Kuo TT, Boyd K, Wang Y, Vignali KM, Cross R, Sehy D, Blumberg RS and Vignali DA: the inhibitory cytokine IL-35 contributes to regulatory T-cell function. Nature 450: 566-569, 2007.

16. Tsuda M, Zhang W, Yang GX, Tsuneyama K, Ando Y, Kawata K, Park O, Leung PS, Coppel RL, Ansari AA, et al: Deletion of interleukin (IL)-12p35 induces liver fibrosis in dominant-negative TGF $\beta$ receptor type II mice. Hepatology 57: 806-816, 2013.

17. Wirtz S, Billmeier U, Mchedlidze T, Blumberg RS and Neurath MF: Interleukin-35 mediates mucosal immune responses that protect against T-cell-dependent colitis. Gastroenterology 141: 1875-1886, 2011.

18. Guo H, Wang W, Zhao N, He X, Zhu L and Jiang X: Inhibiting cardiac allograft rejection with interleukin-35 therapy combined with decitabine treatment in mice. Transpl Immunol 29: 99-104, 2013.

19. Jin E, Wang C, Hu Q, Jin G and Li S: The regular distribution and expression pattern of immunosuppressive cytokine IL-35 in mouse uterus during early pregnancy. Rom J Morphol Embryol 55: 1353-1361, 2014.

20. Ali TK, Matragoon S, Pillai BA, Liou GI and El-Remessy AB: Peroxynitrite mediates retinal neurodegeneration by inhibiting nerve growth factor survival signaling in experimental and human diabetes. Diabetes 57: 889-898, 2008.

21. Niedbala W, Wei XQ, Cai B, Hueber AJ, Leung BP, McInnes IB and Liew FY: IL-35 is a novel cytokine with therapeutic effects against collagen-induced arthritis through the expansion of regulatory T cells and suppression of Th17 cells. Eur J Immunol 37: 3021-3029, 2007.

22. Hori S, Nomura T and Sakaguchi S: Control of regulatory T cell development by the transcription factor Foxp3. Science 299: 1057-1061, 2003.

23. He Y, Jie Y, Wang B, Zeng H, Zhang Y and Pan Z: Adoptive transfer of donor corneal antigen-specific regulatory $\mathrm{T}$ cells can prolong mice corneal grafts survival. Cornea 29 (Suppl 1): S25-S31, 2010.

24. Rossmanith W, Chabicovsky M, Herkner $\mathrm{K}$ and Schulte-Hermann R: Cellular gene dose and kinetics of gene expression in mouse livers transfected by high-volume tail-vein injection of naked DNA. DNA Cell Biol 21: 847-853, 2002.

25. Maruyama H, Higuchi N, Nishikawa Y, Kameda S, Iino N, Kazama JJ, Takahashi N, Sugawa M, Hanawa H, Tada N, et al: High-level expression of naked DNA delivered to rat liver via tail vein injection. J Gene Med 4: 333-341, 2002. 
26. Liu F, Song Y and Liu D: Hydrodynamics-based transfection in animals by systemic administration of plasmid DNA. Gene Ther 6: 1258-1266, 1999.

27. Andrianaivo F, Lecocq M, Wattiaux-De Coninck S, Wattiaux R and Jadot M: Hydrodynamics-based transfection of the liver: entrance into hepatocytes of DNA that causes expression takes place very early after injection. J Gene Med 6: 877-883, 2004.

28. Jiang Y, Zhang Q and Steinle JJ: Intravitreal injection of IGFBP-3 restores normal insulin signaling in diabetic rat retina. PLoS One 9: e93788, 2014.

29. Yoon KC, Ahn KY, Lee JH, Chun BJ, Park SW, Seo MS, Park YG and Kim KK: Lipid-mediated delivery of brain-specific angiogenesis inhibitor 1 gene reduces corneal neovascularization in an in vivo rabbit model. Gene Ther 12: 617-624, 2005.

30. Sonoda S, Tachibana K, Uchino E, Okubo A, Yamamoto M, Sakoda K, Hisatomi T, Sonoda KH, Negishi Y, Izumi Y, et al: Gene transfer to corneal epithelium and keratocytes mediated by ultrasound with microbubbles. Invest Ophthalmol Vis Sci 47: 558-564, 2006

31. Masli S, Turpie B, Hecker KH and Streilein JW: Expression of thrombospondin in TGFbeta-treated APCs and its relevance to their immune deviation-promoting properties. J Immunol 168: 2264-2273, 2002.

32. Torres PF, De Vos AF, van der Gaag R, Martins B and Kijlstra A Cytokine mRNA expression during experimental corneal allograft rejection. Exp Eye Res 63: 453-461, 1996.

33. Song SS, Yuan PF, Chen JY, Fu JJ, Wu HX, Lu JT and Wei W: TGF- $\beta$ favors bone marrow-derived dendritic cells to acquire tolerogenic properties. Immunol Invest 43: 360-369, 2014.

34. Zhou L, Zhu X, Tan J, Wang J and Xing Y: Effect of recombinant adeno-associated virus mediated transforming growth factor-betal on corneal allograft survival after high-risk penetrating keratoplasty. Transpl Immunol 28: 164-169, 2013.

35. Enzmann V, Hollborn M, Wiedemann P and Kohen L: Minor influence of the immunosuppressive cytokines IL-10 and TGF-beta on the proliferation and apoptosis of human retinal pigment epithelial (RPE) cells in vitro. Ocul Immunol Inflamm 9: 259-266, 2001

36. Li B, Tian L, Diao Y, Li X, Zhao L and Wang X: Exogenous IL-10 induces corneal transplantation immune tolerance by a mechanism associated with the altered Th1/Th2 cytokine ratio and the increased expression of TGF- $\beta$. Mol Med Rep 9: 2245-2250, 2014.
37. Klebe S, Sykes PJ, Coster DJ, Krishnan R and Williams KA: Prolongation of sheep corneal allograft survival by ex vivo transfer of the gene encoding interleukin-10. Transplantation 71: 1214-1220, 2001

38. Wang X, Wang W, Xu J, Wu S and Le Q: All-trans retinoid acid promotes allogeneic corneal graft survival in mice by regulating Treg-Th17 balance in the presence of TGF- $\beta$. BMC Immunol 16 : $17,2015$.

39. Klebe S, Coster DJ, Sykes PJ, Swinburne S, Hallsworth P, Scheerlinck JP, Krishnan R and Williams KA: Prolongation of sheep corneal allograft survival by transfer of the gene encoding ovine IL-12-p40 but not IL-4 to donor corneal endothelium. J Immunol 175: 2219-2226, 2005.

40. Gee K, Guzzo C, Che Mat NF, Ma W and Kumar A: The IL-12 family of cytokines in infection, inflammation and autoimmune disorders. Inflamm Allergy Drug Targets 8: 40-52, 2009.

41. King WJ, Comer RM, Hudde T, Larkin DF and George AJ: Cytokine and chemokine expression kinetics after corneal transplantation. Transplantation 70: 1225-1233, 2000.

42. Maier P, Heizmann U, Böhringer D, Kern Y and Reinhard T: Predicting the risk for corneal graft rejection by aqueous humor analysis. Mol Vis 17: 1016-1023, 2011.

43. Park H, Li Z, Yang XO, Chang SH, Nurieva R, Wang $\mathrm{YH}$, Wang Y, Hood L, Zhu Z, Tian Q and Dong C: A distinct lineage of CD 4 T cells regulates tissue inflammation by producing interleukin 17. Nat Immunol 6: 1133-1141, 2005.

44. Tang JL, Subbotin VM, Antonysamy MA, Troutt AB, Rao AS and Thomson AW: Interleukin-17 antagonism inhibits acute but not chronic vascular rejection. Transplantation 72: 348-350, 2001.

45. Antonysamy MA, Fanslow WC, Fu F, Li W, Qian S, Troutt AB and Thomson AW: Evidence for a role of IL-17 in alloimmunity: a novel IL-17 antagonist promotes heart graft survival. Transplant Proc 31: 93, 1999.

46. Chen X, Zhao S, Tang X, Ge H and Liu P: Neutralization of mouse interleukin-17 bioactivity inhibits corneal allograft rejection. Mol Vis 17: 2148-2156, 2011.

47. Chen H, Wang W, Xie H, Xu X, Wu J, Jiang Z, Zhang M, Zhou L and Zheng S: A pathogenic role of IL-17 at the early stage of corneal allograft rejection. Transpl Immunol 21: 155-161, 2009. 